\title{
A Kastler-Kalau-Walze Type Theorem and the Spectral Action for Perturbations of Dirac Operators on Manifolds with Boundary
}

\author{
Yong Wang \\ School of Mathematics and Statistics, Northeast Normal University, Changchun, Jilin 130024, China \\ Correspondence should be addressed to Yong Wang; wangy581@nenu.edu.cn
}

Received 23 October 2013; Accepted 13 January 2014; Published 17 March 2014

Academic Editor: Jaume Giné

Copyright (C) 2014 Yong Wang. This is an open access article distributed under the Creative Commons Attribution License, which permits unrestricted use, distribution, and reproduction in any medium, provided the original work is properly cited.

We prove a Kastler-Kalau-Walze type theorem for perturbations of Dirac operators on compact manifolds with or without boundary. As a corollary, we give two kinds of operator-theoretic explanations of the gravitational action on boundary. We also compute the spectral action for Dirac operators with two-form perturbations on 4-dimensional compact manifolds.

\section{Introduction}

The noncommutative residue found in $[1,2]$ plays a prominent role in noncommutative geometry. In [3], Connes used the noncommutative residue to derive a conformal 4dimensional Polyakov action analogy. In [4], Connes proved that the noncommutative residue on a compact manifold $M$ coincided with Dixmier's trace on pseudodifferential operators of order $-\operatorname{dim} M$. Several years ago, Connes made a challenging observation that the noncommutative residue of the square of the inverse of the Dirac operator was proportional to the Einstein-Hilbert action, which is called the Kastler-Kalau-Walze theorem now. In [5], Kastler gave a brute-force proof of this theorem. In [6], Kalau and Walze proved this theorem by the normal coordinates way simultaneously. In [7], Ackermann gave a note on a new proof of this theorem by the heat kernel expansion way. The KastlerKalau-Walze theorem had been generalized to some cases, for example, Dirac operators with torsion [8], CR manifolds [9], and $\mathbb{R}^{n}[10]$ (see also $[11,12]$ ).

On the other hand, Fedosov et al. defined a noncommutative residue on Boutet de Monvel's algebra and proved that it was the unique continuous trace in [13]. In [14], Schrohe gave the relation between the Dixmier trace and the noncommutative residue for manifolds with boundary. In $[15,16]$, we gave an operator-theoretic explanation of the gravitational action for manifolds with boundary and proved a KastlerKalau-Walze type theorem for Dirac operators and signature operators on manifolds with boundary.

Perturbations of Dirac operators were investigated by several authors. In [17], Sitarz and Zajac investigated the spectral action for scalar perturbations of Dirac operators. In [18, p. 305], Iochum and Levy computed the heat kernel coefficients for Dirac operators with one-form perturbations. In [19], Hanisch et al. derived a formula for the gravitational part of the spectral action for Dirac operators on 4-dimensional spin manifolds with totally antisymmetric torsion and this is a perturbation with three forms of Dirac operators. On the other hand, in [20], Connes and Moscovici considered the conformal perturbations of Dirac operators. Investigating the perturbations of Dirac operators has some significance (see $[18,19,21]$ ). Motivated by [17-19], we study the Dirac operators with general form perturbations. We prove a Kastler-Kalau-Walze type theorem for general form perturbations and the conformal perturbations of Dirac operators for compact manifolds with or without boundary. We also compute the spectral action for Dirac operators with two-form perturbations on 4-dimensional compact manifolds and give detailed computations of spectral action for scalar perturbations of Dirac operators in [17]. 
This paper is organized as follows. In Section 2, we prove the Lichnerowicz formula for perturbations of Dirac operators and prove a Kastler-Kalau-Walze type theorem for perturbations of Dirac operators on 4-dimensional compact manifolds with or without boundary. In Section 3, we prove a Kastler-Kalau-Walze type theorem for conformal perturbations of Dirac operators on compact manifolds with or without boundary. In Section 4, we compute the spectral action for Dirac operators with scalar and two-form perturbations on 4-dimensional compact manifolds.

\section{A Kastler-Kalau-Walze Type Theorem for Perturbations of Dirac Operators}

2.1. A Kastler-Kalau-Walze Type Theorem for Perturbations of Dirac Operators on Manifolds without Boundary. Let $M$ be a smooth compact Riemannian $n$-dimensional manifold without boundary and let $V$ be a vector bundle on $M$. Recall that a differential operator $P$ is of Laplace type if it has locally the form

$$
P=-\left(g^{i j} \partial_{i} \partial_{j}+A^{i} \partial_{i}+B\right),
$$

where $\partial_{i}$ is a natural local frame on $T M, g_{i, j}=g\left(\partial_{i}, \partial_{j}\right)$ and $\left(g^{i j}\right)_{1 \leq i, j \leq n}$ is the inverse matrix associated with the metric matrix $\left(g_{i, j}\right)_{1 \leq i, j \leq n}$ on $M$, and $A^{i}$ and $B$ are smooth sections of $\operatorname{End}(V)$ on $M$ (endomorphism). If $P$ is a Laplace type operator of the form (1), then (see [22]) there is a unique connection $\nabla$ on $V$ and a unique endomorphism $E$ such that

$$
P=-\left[g^{i j}\left(\nabla_{\partial_{i}} \nabla_{\partial_{j}}-\nabla_{\nabla_{\partial_{i}}^{L} \partial_{j}}\right)+E\right],
$$

where $\nabla^{L}$ denotes the Levi-Civita connection on $M$. Moreover (with local frames of $T^{*} M$ and $V$ ), $\nabla_{\partial_{i}}=\partial_{i}+\omega_{i}$ and $E$ are related to $g^{i j}, A^{i}$, and $B$ through

$$
\begin{gathered}
\omega_{i}=\frac{1}{2} g_{i j}\left(A^{j}+g^{k l} \Gamma_{k l}^{j} I d\right), \\
E=B-g^{i j}\left(\partial_{i}\left(\omega_{j}\right)+\omega_{i} \omega_{j}-\omega_{k} \Gamma_{i j}^{k}\right),
\end{gathered}
$$

where $\Gamma_{i j}^{k}$ are the Christoffel coefficients of $\nabla^{L}$.

Now, we let $M$ be an $n$-dimensional oriented spin manifold with Riemannian metric $g$. We recall that the Dirac operator $D$ is locally given as follows in terms of orthonormal frames $e_{i}, 1 \leq i \leq n$, and natural frames $\partial_{i}$ of $T M$ :

$$
D=\sum_{i, j} g^{i j} c\left(\partial_{i}\right) \nabla_{\partial_{j}}^{S}=\sum_{i} c\left(e_{i}\right) \nabla_{e_{i}}^{S}
$$

where $c\left(e_{i}\right)$ denotes the Clifford action which satisfies the relation

$$
\begin{gathered}
c\left(e_{i}\right) c\left(e_{j}\right)+c\left(e_{j}\right) c\left(e_{i}\right)=-2 \delta_{i}^{j}, \\
\nabla_{\partial_{i}}^{S}=\partial_{i}+\sigma_{i}, \\
\sigma_{i}=\frac{1}{4} \sum_{j, k}\left\langle\nabla_{\partial_{i}}^{L} e_{j}, e_{k}\right\rangle c\left(e_{j}\right) c\left(e_{k}\right) .
\end{gathered}
$$

Let

$$
\partial^{j}=g^{i j} \partial_{i}, \quad \sigma^{i}=g^{i j} \sigma_{j}, \quad \Gamma^{k}=g^{i j} \Gamma_{i j}^{k} .
$$

By (6a) in [5], we have

$$
\begin{aligned}
D^{2}= & -g^{i j} \partial_{i} \partial_{j}-2 \sigma^{j} \partial_{j}+\Gamma^{k} \partial_{k} \\
& -g^{i j}\left[\partial_{i}\left(\sigma_{j}\right)+\sigma_{i} \sigma_{j}-\Gamma_{i j}^{k} \sigma_{k}\right]+\frac{1}{4} s,
\end{aligned}
$$

where $s$ is the scalar curvature. Let $\Psi$ be a smooth differential form on $M$ and we also denote the associated Clifford action by $\Psi$. We will compute $D_{\Psi}^{2}:=(D+\Psi)^{2}$. We note that

$$
\begin{gathered}
(D+\Psi)^{2}=D^{2}+D \Psi+\Psi D+\Psi^{2}, \\
D \Psi+\Psi D=\sum_{i j} g^{i j}\left(c\left(\partial_{i}\right) \Psi+\Psi c\left(\partial_{i}\right)\right) \partial_{j} \\
+\sum_{i j} g^{i j}\left(c\left(\partial_{i}\right) \partial_{j}(\Psi)+c\left(\partial_{i}\right) \sigma_{j} \Psi\right. \\
\left.+\Psi c\left(\partial_{i}\right) \sigma_{j}\right) .
\end{gathered}
$$

By (7)-(9), we have

$$
\begin{gathered}
D_{\Psi}^{2}=-g^{i j} \partial_{i} \partial_{j}+\left(-2 \sigma^{j}+\Gamma^{j}+c\left(\partial^{j}\right) \Psi+\Psi c\left(\partial^{j}\right)\right) \partial_{j} \\
+g^{i j}\left[-\partial_{i}\left(\sigma_{j}\right)-\sigma_{i} \sigma_{j}+\Gamma_{i j}^{k} \sigma_{k}+c\left(\partial_{i}\right) \partial_{j}(\Psi)\right. \\
\left.+c\left(\partial_{i}\right) \sigma_{j} \Psi+\Psi c\left(\partial_{i}\right) \sigma_{j}\right]+\frac{1}{4} s+\Psi^{2} .
\end{gathered}
$$

By (10) and (3), we have

$$
\begin{gathered}
\omega_{i}=\sigma_{i}-\frac{1}{2}\left[c\left(\partial_{i}\right) \Psi+\Psi c\left(\partial_{i}\right)\right], \\
E=-c\left(\partial_{i}\right) \partial^{i}(\Psi)-c\left(\partial_{i}\right) \sigma^{i} \Psi-\Psi c\left(\partial_{i}\right) \sigma^{i} \\
-\frac{1}{4} s-\Psi^{2}+\frac{1}{2} \partial^{j}\left[c\left(\partial_{j}\right) \Psi+\Psi c\left(\partial_{j}\right)\right] \\
-\frac{1}{2} \Gamma^{k}\left[c\left(\partial_{k}\right) \Psi+\Psi c\left(\partial_{k}\right)\right]+\frac{1}{2} \sigma^{j}\left[c\left(\partial_{j}\right) \Psi+\Psi c\left(\partial_{j}\right)\right] \\
+\frac{1}{2}\left[c\left(\partial_{j}\right) \Psi+\Psi c\left(\partial_{j}\right)\right] \sigma^{j} \\
-\frac{g^{i j}}{4}\left[c\left(\partial_{i}\right) \Psi+\Psi c\left(\partial_{i}\right)\right]\left[c\left(\partial_{j}\right) \Psi+\Psi c\left(\partial_{j}\right)\right] .
\end{gathered}
$$

For a smooth vector field $X$ on $M$, let $c(X)$ denote the Clifford action. So,

$$
\nabla_{X}=\nabla_{X}^{S}-\frac{1}{2}[c(X) \Psi+\Psi c(X)] .
$$

Since $E$ is globally defined on $M$, so we can perform computations of $E$ in normal coordinates. Taking normal 
coordinates about $x_{0}$, then, $\sigma^{i}\left(x_{0}\right)=0, \partial^{j}\left[c\left(\partial_{j}\right)\right]\left(x_{0}\right)=$ $0, \Gamma^{k}\left(x_{0}\right)=0, g^{i j}\left(x_{0}\right)=\delta_{i}^{j}$, so that

$$
\begin{aligned}
E\left(x_{0}\right)= & -\frac{1}{4} s-\Psi^{2}+\frac{1}{2}\left[\partial^{j}(\Psi) c\left(\partial_{j}\right)-c\left(\partial_{j}\right) \partial^{j}(\Psi)\right] \\
& -\frac{1}{4}\left[c\left(\partial_{i}\right) \Psi+\Psi c\left(\partial_{i}\right)\right]^{2}\left(x_{0}\right) \\
= & -\frac{1}{4} s-\Psi^{2}+\frac{1}{2}\left[e_{j}(\Psi) c\left(e_{j}\right)-c\left(e_{j}\right) e_{j}(\Psi)\right] \\
& -\frac{1}{4}\left[c\left(e_{i}\right) \Psi+\Psi c\left(e_{i}\right)\right]^{2}\left(x_{0}\right) \\
= & -\frac{1}{4} s-\Psi^{2}+\frac{1}{2}\left[\nabla_{e_{j}}^{S}(\Psi) c\left(e_{j}\right)-c\left(e_{j}\right) \nabla_{e_{j}}^{S}(\Psi)\right] \\
& -\frac{1}{4}\left[c\left(e_{i}\right) \Psi+\Psi c\left(e_{i}\right)\right]^{2}\left(x_{0}\right) .
\end{aligned}
$$

We get the following Lichnerowicz formula.

Proposition 1. Let $\Psi$ be a smooth differential form on $M$ and $D_{\Psi}:=D+\Psi$; then

$$
\begin{aligned}
D_{\Psi}^{2}= & -\left[g^{i j}\left(\nabla_{\partial_{i}} \nabla_{\partial_{j}}-\nabla_{\nabla_{\partial_{i}}^{L} \partial_{j}}\right)\right]+\frac{1}{4} s+\Psi^{2} \\
& -\frac{1}{2}\left[\nabla_{e_{j}}^{S}(\Psi) c\left(e_{j}\right)-c\left(e_{j}\right) \nabla_{e_{j}}^{S}(\Psi)\right] \\
& +\frac{1}{4}\left[c\left(e_{i}\right) \Psi+\Psi c\left(e_{i}\right)\right]^{2},
\end{aligned}
$$

where $\nabla_{\partial_{i}}$ is defined by (12) and $X=\partial_{i}$.

We see two special cases of Proposition 1. When $\Psi=f$, where $f$ is a smooth function on $M$, we have

$$
\nabla_{X}=\nabla_{X}^{S}-f_{\mathcal{C}}(X), \quad E=-\frac{1}{4} s+(n-1) f^{2} .
$$

Corollary 2. When $\Psi=f$, one has

$$
D_{f}^{2}=-\left[g^{i j}\left(\nabla_{\partial_{i}} \nabla_{\partial_{j}}-\nabla_{\nabla_{\partial_{i}}^{L} \partial_{j}}\right)\right]+\frac{1}{4} s+(1-n) f^{2}
$$

Let $\eta=a_{i} e^{i}$ be a one form, where $a_{i}$ is a smooth real function, let $e^{i}$ be a dual orthonormal frame by parallel transport along geodesic, and let $X=a_{i} e_{i}$ be the dual vector field of $\eta$. When $\Psi=\sqrt{-1} c(\eta)$, by (12), we have
$\nabla_{Y}=\nabla_{Y}^{S}+\sqrt{-1} g(X, Y)$, where $Y$ is a smooth vector field on $M$. By $e_{j}\left(c\left(e_{i}\right)\right)=0$ and $d e^{l}\left(x_{0}\right)=0$ (see [15]), we have

$$
\begin{aligned}
E\left(x_{0}\right)= & -\frac{1}{4} s-|X|^{2}+\frac{\sqrt{-1}}{2} \\
& \times\left[e_{j}\left(a^{k}\right) c\left(e_{k}\right) c\left(e_{j}\right)-c\left(e_{j}\right) c\left(e_{k}\right) e_{j}\left(a^{k}\right)\right] \\
& +\frac{1}{4}\left[c\left(e_{i}\right) c(X)+c(X) c\left(e_{i}\right)\right]^{2} \\
= & -\frac{1}{4} s+\frac{\sqrt{-1}}{2} e_{j}\left(a^{k}\right)\left[c\left(e_{k}\right) c\left(e_{j}\right)-c\left(e_{j}\right) c\left(e_{k}\right)\right] \\
= & -\frac{1}{4} s+\sqrt{-1} \sum_{k \neq j} e_{j}\left(a^{k}\right) c\left(e_{k}\right) c\left(e_{j}\right)\left(x_{0}\right) \\
= & -\frac{1}{4} s-\sqrt{-1} c(d \eta)\left(x_{0}\right) .
\end{aligned}
$$

Corollary 3. For a one-form $\eta$ and the Clifford action $c(\eta)$, one has

$$
\begin{aligned}
(D+\sqrt{-1} c(\eta))^{2}= & -\left[g^{i j}\left(\nabla_{\partial_{i}} \nabla_{\partial_{j}}-\nabla_{\nabla_{\partial_{i}}^{L} \partial_{j}}\right)\right] \\
& +\frac{1}{4} s+\sqrt{-1} c(d \eta) .
\end{aligned}
$$

When $\Psi$ is a two-form, we let $\Psi=2 \sum_{k<l} a_{k l} e^{k} \wedge e^{l}=$ $\sum a_{k l} e^{k} \wedge e^{l}$, where $a_{k l}=-a_{l k}$, and $c(\Psi)=\sum a_{k l} c\left(e_{k}\right) c\left(e_{l}\right)$. So,

$$
\nabla_{e_{i}}=e_{i}+\frac{1}{4} \sum_{s, t} \omega_{s t}\left(e_{i}\right) c\left(e_{s}\right) c\left(e_{t}\right)-\sum_{k, l \neq i} a_{k l} c\left(e_{k}\right) c\left(e_{l}\right) c\left(e_{i}\right),
$$

where $\omega_{s t}\left(e_{i}\right)$ denotes the connection coefficient. By (13),

$$
\begin{aligned}
E= & -\frac{1}{4} s-\left[a_{k l} c\left(e_{k}\right) c\left(e_{l}\right)\right]^{2} \\
& +\frac{1}{2}\left\{e_{j}\left(a_{k l}\right)\left[c\left(e_{k}\right) c\left(e_{l}\right) c\left(e_{j}\right)-c\left(e_{j}\right) c\left(e_{k}\right) c\left(e_{l}\right)\right]\right\} \\
& -\frac{1}{4}\left[a_{k l}\left(c\left(e_{i}\right) c\left(e_{k}\right) c\left(e_{l}\right)+c\left(e_{k}\right) c\left(e_{l}\right) c\left(e_{i}\right)\right)\right]^{2} .
\end{aligned}
$$

Let $S(T M)$ be the spinor bundle on $M$ and $\operatorname{dim}(S(T M))=d$ and $\operatorname{Tr}(A)$ denote the trace of $A$, for $A \in \operatorname{End}(S(T M))$. Since, for $k \neq l, \widetilde{k} \neq \widetilde{l}$,

$$
\operatorname{Tr}\left[c\left(e_{k}\right) c\left(e_{l}\right) c\left(e_{\tilde{k}}\right) c\left(e_{\bar{l}}\right)\right]=d\left(-\delta_{k}^{\tilde{k}} \delta_{l}^{\widetilde{l}}+\delta_{k}^{\widetilde{l}} \delta_{l}^{\tilde{k}}\right),
$$

we have

$$
\operatorname{Tr}\left\{\left[a_{k l} c\left(e_{k}\right) c\left(e_{l}\right)\right]^{2}\right\}=-2 d a_{k l}^{2}
$$


Since the trace of the product of odd Clifford elements is zero, we have

$$
\begin{aligned}
& \operatorname{Tr}\left[\frac{1}{2}\left\{e_{j}\left(a_{k l}\right)\left[c\left(e_{k}\right) c\left(e_{l}\right) c\left(e_{j}\right)-c\left(e_{j}\right) c\left(e_{k}\right) c\left(e_{l}\right)\right]\right\}\right]=0 \\
& \operatorname{Tr}\left\{\left[a_{k l}\left(c\left(e_{i}\right) c\left(e_{k}\right) c\left(e_{l}\right)+c\left(e_{k}\right) c\left(e_{l}\right) c\left(e_{i}\right)\right)\right]^{2}\right\} \\
& =a_{k l} a_{\widetilde{k l}} \operatorname{Tr}\left[c\left(e_{k}\right) c\left(e_{l}\right) c\left(e_{\widetilde{k}}\right) c\left(e_{\tilde{l}}\right) c\left(e_{i}\right)^{2}\right. \\
& +c\left(e_{k}\right) c\left(e_{l}\right) c\left(e_{i}\right)^{2} c\left(e_{\widetilde{k}}\right) c\left(e_{\widetilde{l}}\right) \\
& +c\left(e_{i}\right) c\left(e_{k}\right) c\left(e_{l}\right) c\left(e_{i}\right) c\left(e_{\tilde{k}}\right) c\left(e_{\tilde{l}}\right) \\
& \left.+c\left(e_{k}\right) c\left(e_{l}\right) c\left(e_{i}\right) c\left(e_{\widetilde{k}}\right) c\left(e_{\tilde{l}}\right) c\left(e_{i}\right)\right] \\
& =-2 n d a_{k l} a_{\widetilde{k l}}\left(-\delta_{k}^{\tilde{k}} \delta_{l}^{\tilde{l}}+\delta_{k}^{\tilde{l}} \delta_{l}^{\tilde{k}}\right) \\
& -2 \sum_{i \neq k, l} a_{k l} a_{\widetilde{k l}} \operatorname{Tr}\left[c\left(e_{k}\right) c\left(e_{l}\right) c\left(e_{\widetilde{k}}\right) c\left(e_{\tilde{l}}\right)\right] \\
& +2 \sum_{i=k} a_{k l} a_{\widetilde{k l}} \operatorname{Tr}\left[c\left(e_{k}\right) c\left(e_{l}\right) c\left(e_{\tilde{k}}\right) c\left(e_{\tilde{l}}\right)\right] \\
& +2 \sum_{i=l} a_{k l} a_{\widetilde{k l}} \operatorname{Tr}\left[c\left(e_{k}\right) c\left(e_{l}\right) c\left(e_{\widetilde{k}}\right) c\left(e_{\tilde{l}}\right)\right] \\
& =4 n d a_{k l}^{2}+(n-2) 4 d a_{k l}^{2}-4 d a_{k l}^{2}-4 d a_{k l}^{2} \\
& =8(n-2) d a_{k l}^{2} \text {. }
\end{aligned}
$$

By (20) and (22)-(24), we have

$$
\operatorname{Tr} E=d\left(-\frac{1}{4} s+(6-2 n)|\Psi|^{2}\right)
$$

and we get the following.

Corollary 4. Let $\Psi=\sum a_{k l} e^{k} \wedge e^{l}$ and $a_{k l}=-a_{l k}$; then $\operatorname{tr} E=$ $d\left(-(1 / 4) s+(6-2 n)|\Psi|^{2}\right)$.

For a general differential form $\Psi$, by $(13)$ and $\operatorname{Tr}(A B)=$ $\operatorname{Tr}(B A)$, we have

$$
\begin{aligned}
\operatorname{Tr}(E) & =\operatorname{Tr}\left[-\frac{1}{4} s-\Psi^{2}-\frac{1}{4}\left[c\left(e_{i}\right) \Psi+\Psi_{c}\left(e_{i}\right)\right]^{2}\right] \\
& =\operatorname{Tr}\left[-\frac{1}{4} s-\frac{1}{2} \Psi c\left(e_{i}\right) \Psi_{c}\left(e_{i}\right)+\left(\frac{n}{2}-1\right) \Psi^{2}\right] .
\end{aligned}
$$

By the Kastler-Kalau-Walze theorem (see $[5,6]$ ), we have

$$
\operatorname{Wres}\left(D_{\Psi}^{-n+2}\right)=\frac{(2 \pi)^{n / 2}}{(n / 2-2) !} \int_{M} \operatorname{Tr}\left[\frac{1}{6} s+E\right] d v o l_{M},
$$

where Wres denotes the noncommutative residue (see [2]). By (26) and (27), we have the following.
Theorem 5. For even n-dimensional compact spin manifolds without boundary and a general form $\Psi$, the following equality holds:

$$
\begin{aligned}
& \text { Wres }\left(D_{\Psi}^{-n+2}\right) \\
& \begin{array}{r}
=\frac{(2 \pi)^{n / 2}}{(n / 2-2) !} \\
\times \int_{M} \operatorname{Tr}\left[-\frac{1}{12} s\right. \\
\quad-\frac{1}{2} c(\Psi) c\left(e_{i}\right) c(\Psi) c\left(e_{i}\right) \\
\left.\quad+(n / 2-1) c(\Psi)^{2}\right] d v o l_{M} .
\end{array}
\end{aligned}
$$

By Corollary 2, we have the following.

Corollary 6. For even $n$-dimensional compact spin manifolds without boundary and a smooth function $f$ on $M$, the following equality holds:

Wres $\left(D_{f}^{-n+2}\right)=\frac{(2 \pi)^{n / 2} d}{(n / 2-2) !} \int_{M}\left[-\frac{1}{12} s+(n-1) f^{2}\right] d v_{M} l_{M}$.

By Corollary 3, we have the following.

Corollary 7. For even n-dimensional compact spin manifolds without boundary and a one-form $\Psi$, the following equality holds:

$$
\text { Wres }\left(D_{\Psi}^{-n+2}\right)=-\frac{(2 \pi)^{n / 2} d}{12 \times(n / 2-2) !} \int_{M} s d v o l_{M} \text {. }
$$

By Corollary 4 and (27), we have the following.

Corollary 8. For even n-dimensional compact spin manifolds without boundary and a two-form $\Psi$, the following equality holds:

$$
\text { Wres } \begin{aligned}
\left(D_{\Psi}^{-n+2}\right)= & \frac{(2 \pi)^{n / 2} d}{(n / 2-2) !} \\
& \times \int_{M} \operatorname{Tr}\left[-\frac{1}{12} s+(6-2 n)|\Psi|^{2}\right] d v o l_{M}
\end{aligned}
$$

2.2. A Kastler-Kalau-Walze Type Theorem for Perturbations of Dirac Operators on Manifolds with Boundary. We now let $M$ be a compact 4-dimensional spin manifold with boundary $\partial M$ and let $U \subset M$ be the collar neighborhood of $\partial M$ which is diffeomorphic to $\partial M \times[0,1)$. And we will compute the noncommutative residue for manifolds with boundary of $\left(\pi^{+} D_{\Psi}^{-1}\right)^{2}$. That is, we will compute $\overparen{\text { Wres }}\left[\left(\pi^{+} D_{\Psi}^{-1}\right)^{2}\right]$ (for the related definitions, see [15]) and we take the metric as in [15]. 
Let $\left(x^{\prime}, x_{n}\right) \in U$, where $x^{\prime} \in \partial M$ and $x_{n}$ denotes the normal direction coordinate. By (2.2.4) in [15], we have

$$
\begin{aligned}
\widetilde{\text { Wres }} & {\left[\left(\pi^{+} D_{\Psi}^{-1}\right)^{2}\right] } \\
= & \int_{M} \int_{|\xi|=1} \operatorname{trace}_{S(T M)}\left[\sigma_{-4}\left(D_{\Psi}^{-2}\right)\right] \sigma(\xi) d x \\
& +\int_{\partial M} \Phi
\end{aligned}
$$

where

$\Phi$

$$
\begin{aligned}
=\int_{\left|\xi^{\prime}\right|=1} \int_{-\infty}^{+\infty} \sum_{j, k=0}^{\infty} \sum \frac{(-i)^{|\alpha|+j+k+1}}{\alpha !(j+k+1) !} \\
\times \operatorname{trace}_{S(T M)}\left[\partial_{x_{n}}^{j} \partial_{\xi^{\prime}}^{\alpha} \partial_{\xi_{n}}^{k} \sigma_{r}^{+}\left(D_{\Psi}^{-1}\right)\left(x^{\prime}, 0, \xi^{\prime}, \xi_{n}\right)\right. \\
\times \partial_{x^{\prime}}^{\alpha} \partial_{\xi_{n}}^{j+1} \partial_{x_{n}}^{k} \sigma_{l}\left(D_{\Psi}^{-1}\right) \\
\left.\times\left(x^{\prime}, 0, \xi^{\prime}, \xi_{n}\right)\right] d \xi_{n} \sigma\left(\xi^{\prime}\right) d x^{\prime},
\end{aligned}
$$

where the sum is taken over $r-k-|\alpha|+l-j-1=-4, r, l \leq-1$, and $\sigma_{r}^{+}\left(D_{\Psi}^{-1}\right)=\pi_{\xi_{n}}^{+} \sigma_{r}\left(D_{\Psi}^{-1}\right)$ (for the definition of $\pi^{+}$, see [15]). By Theorem 5, we have

$$
\begin{aligned}
\int_{M} \int_{|\xi|=1} \operatorname{tr}\left[\sigma_{-4}\left(D_{\Psi}^{-2}\right)\right] \sigma(\xi) d x \\
=4 \pi^{2} \int_{M} \operatorname{Tr}\left[-\frac{1}{12} s-\frac{1}{2} \Psi c\left(e_{i}\right) \Psi c\left(e_{i}\right)\right. \\
\left.+\left(\frac{n}{2}-1\right) \Psi^{2}\right] d v o l_{M} .
\end{aligned}
$$

So, we only need to compute $\int_{\partial M} \Phi$. In analogy with Lemma 2.1 of [15], we can prove the following useful result.

Lemma 9. The symbolic calculus of pseudodifferential operators yields

$$
\begin{gathered}
q_{-1}\left(D_{\Psi}^{-1}\right)=\frac{\sqrt{-1} c(\xi)}{|\xi|^{2}} \\
q_{-2}\left(D_{\Psi}^{-1}\right)=q_{-2}\left(D^{-1}\right)+\frac{c(\xi) \Psi c(\xi)}{|\xi|^{4}} .
\end{gathered}
$$

Similar to the computations in Section 2.2.2 in [15], we can split $\Phi$ into the sum of five terms. Since $q_{-1}\left(D_{\Psi}^{-1}\right)=$ $q_{-1}\left(D^{-1}\right)$, then terms (a) (I), (a) (II), and (a) (III) in our case are the same as the terms (a) (I), (a) (II), and (a) (III) in [15], so

term (a) (I)

$$
\begin{aligned}
=-\int_{\left|\xi^{\prime}\right|=1} \int_{-\infty}^{+\infty} \sum_{|\alpha|=1} \operatorname{trace} & {\left[\partial_{\xi^{\prime}}^{\alpha} \pi_{\xi_{n}}^{+} q_{-1}\right.} \\
& \left.\times \partial_{x^{\prime}}^{\alpha} \partial_{\xi_{n}} q_{-1}\right] \\
& \times\left(x_{0}\right) d \xi_{n} \sigma\left(\xi^{\prime}\right) d x^{\prime}
\end{aligned}
$$

$=0$,

$\operatorname{term}(\mathrm{a})(\mathrm{II})$

$$
\begin{gathered}
=-\frac{1}{2} \int_{\left|\xi^{\prime}\right|=1} \int_{-\infty}^{+\infty} \operatorname{trace}\left[\partial_{x_{n}} \pi_{\xi_{n}}^{+} q_{-1}\right. \\
\left.\times \partial_{\xi_{n}}^{2} q_{-1}\right] \\
\times\left(x_{0}\right) d \xi_{n} \sigma\left(\xi^{\prime}\right) d x^{\prime} \\
=-\frac{3}{8} \pi h^{\prime}(0) \Omega_{3} d x^{\prime},
\end{gathered}
$$

term (a) (III)

$$
\begin{aligned}
& =-\frac{1}{2} \int_{\left|\xi^{\prime}\right|=1} \int_{-\infty}^{+\infty} \operatorname{trace}\left[\partial_{\xi_{n}} \pi_{\xi_{n}}^{+} q_{-1}\right. \\
& \left.\times \partial_{\xi_{n}} \partial_{x_{n}} q_{-1}\right] \\
& \times\left(x_{0}\right) d \xi_{n} \sigma\left(\xi^{\prime}\right) d x^{\prime} \\
& =\frac{3}{8} \pi h^{\prime}(0) \Omega_{3} d x^{\prime} .
\end{aligned}
$$

Then, we only need to compute term (b) and term (c). By Lemma 9,

term (b)

$$
\begin{aligned}
&:=-i \int_{\left|\xi^{\prime}\right|=1} \int_{-\infty}^{+\infty} \operatorname{trace}[ \pi_{\xi_{n}}^{+} q_{-2}\left(D^{-1}\right) \\
&\left.\times \partial_{\xi_{n}} q_{-1}\left(D^{-1}\right)\right] \\
& \times\left(x_{0}\right) d \xi_{n} \sigma\left(\xi^{\prime}\right) d x^{\prime} \\
&-i \int_{\left|\xi^{\prime}\right|=1} \int_{-\infty}^{+\infty} \operatorname{trace}\left[\pi_{\xi_{n}}^{+}\left(\frac{c(\xi) \Psi c(\xi)}{|\xi|^{4}}\right)\right. \\
&\left.\times \partial_{\xi_{n}} q_{-1}\left(D^{-1}\right)\right] \\
& \times\left(x_{0}\right) d \xi_{n} \sigma\left(\xi^{\prime}\right) d x^{\prime} .
\end{aligned}
$$


By term (b) in [15], we have

$$
\begin{gathered}
-i \int_{\left|\xi^{\prime}\right|=1} \int_{-\infty}^{+\infty} \operatorname{trace}\left[\pi_{\xi_{n}}^{+} q_{-2}\left(D^{-1}\right)\right. \\
\left.\times \partial_{\xi_{n}} q_{-1}\left(D^{-1}\right)\right] \\
\times\left(x_{0}\right) d \xi_{n} \sigma\left(\xi^{\prime}\right) d x^{\prime} \\
=\frac{9}{8} \pi h^{\prime}(0) \Omega_{3} d x^{\prime}
\end{gathered}
$$

where $\Omega_{3}$ is the canonical volume of 3-dimensional unit sphere. Moreover,

$$
\begin{aligned}
& \left.\pi_{\xi_{n}}^{+}\left(\frac{c(\xi) \Psi c(\xi)}{|\xi|^{4}}\right)\left(x_{0}\right)\right|_{\left|\xi^{\prime}\right|=1} \\
& =\pi_{\xi_{n}}^{+}\left[\frac{\left[c\left(\xi^{\prime}\right)+\xi_{n} c\left(d x_{n}\right)\right] \Psi\left[c\left(\xi^{\prime}\right)+\xi_{n} c\left(d x_{n}\right)\right]}{\left(1+\xi_{n}^{2}\right)^{2}}\right] \\
& =\frac{1}{2 \pi i} \int_{\Gamma^{+}}\left(\left(\left(c\left(\xi^{\prime}\right) \Psi c\left(\xi^{\prime}\right)+c\left(d x_{n}\right) \Psi c\left(\xi^{\prime}\right) \eta_{n}\right.\right.\right. \\
& +c\left(\xi^{\prime}\right) \Psi_{c}\left(d x_{n}\right) \eta_{n} \\
& \left.+c\left(d x_{n}\right) \Psi c\left(d x_{n}\right) \eta_{n}^{2}\right) \\
& \left.\times\left(\left(\eta_{n}+i\right)^{2}\left(\xi_{n}-\eta_{n}\right)\right)^{-1}\right) \\
& \left.\times\left(\left(\eta_{n}-i\right)^{2}\right)^{-1}\right) d \eta_{n} \\
& =\left[\left(c\left(\xi^{\prime}\right) \Psi_{c}\left(\xi^{\prime}\right)+c\left(d x_{n}\right) \Psi c\left(\xi^{\prime}\right) \eta_{n}\right.\right. \\
& \left.+c\left(\xi^{\prime}\right) \Psi c\left(d x_{n}\right) \eta_{n}+c\left(d x_{n}\right) \Psi c\left(d x_{n}\right) \eta_{n}^{2}\right) \\
& \left.\times\left(\left(\eta_{n}+i\right)^{2}\left(\xi_{n}-\eta_{n}\right)\right)^{-1}\right]\left.^{(1)}\right|_{\eta_{n}=i} \\
& =-\frac{i \xi_{n}+2}{4\left(\xi_{n}-i\right)^{2}} c\left(\xi^{\prime}\right) \Psi c\left(\xi^{\prime}\right) \\
& -\frac{i}{4\left(\xi_{n}-i\right)^{2}}\left[c\left(d x_{n}\right) \Psi c\left(\xi^{\prime}\right)+c\left(\xi^{\prime}\right) \Psi c\left(d x_{n}\right)\right] \\
& -\frac{i \xi_{n}}{4\left(\xi_{n}-i\right)^{2}} c\left(d x_{n}\right) \Psi c\left(d x_{n}\right) \\
& \left.\partial_{\xi_{n}} q_{-1}\right|_{\left|\xi^{\prime}\right|=1}=\sqrt{-1}\left[\frac{1-\xi_{n}^{2}}{\left(1+\xi_{n}^{2}\right)^{2}} c\left(d x_{n}\right)-\frac{2 \xi_{n}}{\left(1+\xi_{n}^{2}\right)^{2}} c\left(\xi^{\prime}\right)\right] \text {. }
\end{aligned}
$$

By (39) and

$$
\left.c\left(\xi^{\prime}\right)^{2}\right|_{\left|\xi^{\prime}\right|=1}=-1, \quad c\left(d x_{n}\right)^{2}=-1
$$

$$
c\left(\xi^{\prime}\right) c\left(d x_{n}\right)=-c\left(d x_{n}\right) c\left(\xi^{\prime}\right), \quad \operatorname{Tr}(A B)=\operatorname{Tr}(B A)
$$

we get

$$
\begin{aligned}
\operatorname{trace}[ & \left.\pi_{\xi_{n}}^{+}\left(\frac{c(\xi) \Psi c(\xi)}{|\xi|^{4}}\right) \times \partial_{\xi_{n}} q_{-1}\left(D^{-1}\right)\right]\left.\left(x_{0}\right)\right|_{\left|\xi^{\prime}\right|=1} \\
= & \frac{\sqrt{-1}}{2\left(1+\xi_{n}^{2}\right)^{2}} \operatorname{Tr}\left[c\left(d x_{n}\right) \Psi\right] \\
& +\frac{1}{2\left(1+\xi_{n}^{2}\right)^{2}} \operatorname{Tr}\left[c\left(\xi^{\prime}\right) \Psi\right]
\end{aligned}
$$

Considering, for $i<n, \int_{\left|\xi^{\prime}\right|=1} \xi_{i} \sigma\left(\xi^{\prime}\right)=0$, then

$$
\begin{aligned}
& -i \int_{\left|\xi^{\prime}\right|=1} \int_{-\infty}^{+\infty} \operatorname{trace}\left[\pi_{\xi_{n}}^{+}\left(\frac{c(\xi) \Psi c(\xi)}{|\xi|^{4}}\right)\right. \\
& \left.\times \partial_{\xi_{n}} q_{-1}\left(D^{-1}\right)\right] \\
& \times\left(x_{0}\right) d \xi_{n} \sigma\left(\xi^{\prime}\right) d x^{\prime} \\
& =\frac{\pi}{4} \Omega_{3} \operatorname{Tr}\left[c\left(d x_{n}\right) \Psi\right] d x^{\prime}, \\
& \text { term }(\mathrm{b})=\frac{9}{8} \pi h^{\prime}(0) \Omega_{3} d x^{\prime}+\frac{\pi}{4} \Omega_{3} \operatorname{Tr}\left[c\left(d x_{n}\right) \Psi\right] d x^{\prime} \text {. }
\end{aligned}
$$

Similarly, we have

$$
\text { term }(\mathrm{c})=-\frac{9}{8} \pi h^{\prime}(0) \Omega_{3} d x^{\prime}-\frac{\pi}{4} \Omega_{3} \operatorname{Tr}\left[c\left(d x_{n}\right) \Psi\right] d x^{\prime} .
$$

Then, the sum of terms (b) and (c) is zero and $\Phi$ is zero. Then, we get the following.

Theorem 10. Let $M$ be a 4-dimensional compact spin manifold with boundary $\partial M$ and the metric $g^{M}$ (see (1.3) in [15]). Let $\Psi$ be a general differential form on $M$. Then,

$$
\begin{gathered}
\overparen{\text { Wres }}\left[\left(\pi^{+} D_{\Psi}^{-1}\right)^{2}\right] \\
=4 \pi^{2} \int_{M} \operatorname{Tr}\left[-\frac{1}{12} s-\frac{1}{2} c(\Psi) c\left(e_{i}\right) c(\Psi) c\left(e_{i}\right)\right. \\
\left.+c(\Psi)^{2}\right] d v o l_{M} .
\end{gathered}
$$

In [16], we proved a Kastler-Kalau-Walze theorem associated with Dirac operators for 6-dimensional spin manifolds with boundary. In fact, our computations hold for general Laplacians. This implies the following.

Proposition 11 (see [16]). Let $M$ be a 6-dimensional compact Riemannian manifold with boundary $\partial M$ and the metric $g^{M}$ 
(see (1.3) in [15]). Let $\Delta$ be a general Laplacian acting on sections of the vector bundle $V$. Then,

$$
\widetilde{\text { Wres }}\left[\left(\pi^{+} \Delta^{-1}\right)^{2}\right]=8 \pi^{3} \int_{M} \operatorname{Tr}\left[\frac{s}{6}+E\right] d v o l_{M} \text {. }
$$

Since $D_{\Psi}^{2}$ is a general Laplacian, then we get the following.

Corollary 12. Let $M$ be a 6-dimensional compact spin manifold with boundary $\partial M$ and the metric $g^{M}$. Let $\Psi$ be a general differential form on $M$. Then,

$$
\begin{aligned}
& \text { Wres } \\
& {\left[\left(\pi^{+} D_{\Psi}^{-2}\right)^{2}\right]} \\
& =8 \pi^{3} \int_{M} \operatorname{Tr}\left[-\frac{1}{12} s-\frac{1}{2} \Psi_{c}\left(e_{i}\right) \Psi c\left(e_{i}\right)+2 \Psi^{2}\right] d v o l_{M} .
\end{aligned}
$$

In the above two cases, the boundary terms vanish. In the following, we will give a boundary term nonvanishing case and compute Wres $\left(\left(D_{\Psi} D\right)^{-1}\right)$. We have

$$
\begin{gathered}
D_{\Psi} D=-g^{i j} \partial_{i} \partial_{j}+\left(-2 \sigma^{j}+\Gamma^{j}+\Psi_{c}\left(\partial^{j}\right)\right) \partial_{j} \\
+g^{i j}\left[-\partial_{i}\left(\sigma_{j}\right)-\sigma_{i} \sigma_{j}+\Gamma_{i j}^{k} \sigma_{k}+\Psi_{c}\left(\partial_{i}\right) \sigma_{j}\right] \\
+\frac{1}{4} s, \\
\omega_{i}=\sigma_{i}-\frac{1}{2} \Psi_{c}\left(\partial_{i}\right), \\
-\Psi_{c}\left(\partial_{i}\right) \sigma^{i}-\frac{1}{4} s+\frac{1}{2} \partial^{j}\left[\Psi_{c}\left(\partial_{j}\right)\right]-\frac{1}{2} \Gamma^{k} \Psi_{c}\left(\partial_{k}\right) \\
+g^{i j}\left[\frac{1}{2} \sigma_{i} \Psi_{c}\left(\partial_{j}\right)+\frac{1}{2} \Psi_{c}\left(\partial_{i}\right) \sigma_{j}-\frac{1}{4} \Psi_{c}\left(\partial_{i}\right) \Psi_{c}\left(\partial_{j}\right)\right] .
\end{gathered}
$$

Similar to the proof of (13), we have

$$
E=-\frac{1}{4} s+\frac{1}{2} \nabla_{e_{i}}^{S}(\Psi) c\left(e_{i}\right)-\frac{1}{4} \Psi c\left(e_{i}\right) \Psi c\left(e_{i}\right) .
$$

So,

$$
\begin{aligned}
D_{\Psi} D= & -\left[g^{i j}\left(\nabla_{\partial_{i}} \nabla_{\partial_{j}}-\nabla_{\nabla_{\partial_{i}}^{L} \partial_{j}}\right)\right] \\
& +\frac{1}{4} s-\frac{1}{2} \nabla_{e_{i}}^{S}(\Psi) c\left(e_{i}\right)+\frac{1}{4} \Psi c\left(e_{i}\right) \Psi c\left(e_{i}\right) .
\end{aligned}
$$

Then, we get the following.

Proposition 13. Let $M$ be a 4-dimensional compact spin manifold without boundary. Then,

$$
\begin{aligned}
& \text { Wres }\left[\left(D_{\Psi} D\right)^{-1}\right] \\
& \begin{aligned}
=4 \pi^{2} \int_{M} \operatorname{Tr}[ & -\frac{1}{12} s+\frac{1}{2} \nabla_{e_{i}}^{S}(\Psi) c\left(e_{i}\right) \\
& \left.-\frac{1}{4} \Psi_{c}\left(e_{i}\right) \Psi_{c}\left(e_{i}\right)\right] d v o l_{M} .
\end{aligned}
\end{aligned}
$$

When $\Psi$ is a one-form, we can get the following corollary.

Corollary 14. Let $M$ be a 4-dimensional compact spin manifold without boundary and let $\Psi$ be a one-form on $M$. Then,

$$
\begin{aligned}
& \text { Wres }\left[\left(D_{\Psi} D\right)^{-1}\right] \\
& =16 \pi^{2} \int_{M}\left[-\frac{1}{12} s+\frac{1}{2} \delta(\Psi)-2|\Psi|^{2}\right] d v o l_{M}
\end{aligned}
$$

Now, we compute $\overparen{\text { Wres }}\left[\pi^{+} D_{\Psi}^{-1} \pi^{+} D^{-1}\right]$. We have that terms (a) and (b) are the same as in Theorem 10, and since term $(c)=-(9 / 8) \pi h^{\prime}(0) \Omega_{3} d x^{\prime}$, we get

$$
\int_{\partial M} \Phi=\frac{\pi}{4} \Omega_{3} \int_{\partial M} \operatorname{Tr}\left[c\left(d x_{n}\right) \Psi\right] d v o l_{\partial_{M}}
$$

and the following.

Proposition 15. Let $M$ be a 4-dimensional compact spin manifold with boundary. Then,

$$
\begin{gathered}
\overparen{\text { Wres }}\left[\pi^{+} D_{\Psi}^{-1} \pi^{+} D^{-1}\right] \\
=4 \pi^{2} \int_{M} \operatorname{Tr}\left[-\frac{1}{12} s+\frac{1}{2} e_{i}(\Psi) c\left(e_{i}\right)\right. \\
\left.\quad-\frac{1}{4} \Psi c\left(e_{i}\right) \Psi c\left(e_{i}\right)\right] d v o l_{M} \\
+\frac{\pi}{4} \Omega_{3} \int_{\partial M} \operatorname{Tr}\left[c\left(d x_{n}\right) \Psi\right] d v o l_{\partial_{M} .}
\end{gathered}
$$

Remark 16. When $\Psi$ is not a one-form, then the boundary term vanishes. When $\Psi=K d x_{n}$ near the boundary, where $K$ is the extrinsic curvature, then the boundary term is proportional to the gravitational action on the boundary. In fact, the reason for the boundary term being not zero is that $\pi^{+} D_{\Psi}$ and $\pi^{+} D$ are not symmetric.

\section{A Kastler-Kalau-Walze Type Theorem for Conformal Perturbations of Dirac Operators}

In [20], Connes and Moscovici defined a twisted spectral triple and considered the conformal Dirac operator $e^{h} D e^{h}$, where $h$ is a smooth function on a manifold $M$ without boundary. We want to compute Wres $\left[\left(e^{h} D e^{h}\right)^{-2}\right]$. We know that

$$
\begin{aligned}
\text { Wres }\left[\left(e^{h} D e^{h}\right)^{-2}\right] & =\text { Wres }\left[e^{-h} D^{-1} e^{-2 h} D^{-1} e^{-h}\right] \\
& =\text { Wres }\left[e^{-2 h} D^{-1} e^{-2 h} D^{-1}\right] .
\end{aligned}
$$

In the following, we will compute the more general case, that is, Wres $\left[f D^{-1} g D^{-1}\right]$, for nonzero smooth functions $f$ and $g$, and prove a Kastler-Kalau-Walze type theorem for conformal 
Dirac operators. When $f=g=e^{-2 h}$, we get the expression of Wres $\left[\left(e^{h} D e^{h}\right)^{-2}\right]$. We have

$$
\begin{aligned}
& \text { Wres }\left[f D^{-1} g D^{-1}\right] \\
& =\operatorname{Wres}\left[\left(f^{-1} D g^{-1} D\right)^{-1}\right] \\
& =\operatorname{Wres}\left\{\left(f^{-1} g^{-1} D^{2}+f^{-1}\left[D, g^{-1}\right] D\right)^{-1}\right\} \\
& =\int_{M} f g \text { wres }\left[\left(D^{2}-g^{-1} c(d g) D\right)^{-1}\right],
\end{aligned}
$$

where Wres denotes the residue density, and we note that the Kastler-Kalau-Walze theorem holds at the residue density level. Some computations show that

$$
\begin{aligned}
& D^{2}-g^{-1} c(d g) D \\
& =-g^{i j} \partial_{i} \partial_{j}+\left[-2 \sigma^{j}+\Gamma^{j}-g^{-1} c(d g) c\left(\partial^{j}\right)\right] \partial_{j} \\
& +\left[-\partial^{j} \sigma_{j}-\sigma^{j} \sigma_{j}+\Gamma^{k} \sigma_{k}+\frac{1}{4} s-g^{-1} c(d g) c\left(\partial^{j}\right) \sigma_{j}\right] \\
& \omega_{i}=\sigma_{i}+\frac{1}{2} g^{-1} c(d g) c\left(\partial_{i}\right), \\
& E=-\frac{s}{4}+g^{-1} c(d g) c\left(\partial^{j}\right) \sigma_{j} \\
& -\partial^{j}\left[\frac{1}{2} g^{-1} c(d g) c\left(\partial_{j}\right)\right] \\
& -\frac{1}{2} \sigma^{j} g^{-1} c(d g) c\left(\partial_{j}\right)-\frac{1}{2} g^{-1} c(d g) c\left(\partial_{i}\right) \sigma^{i} \\
& -\frac{1}{4} g^{i j} g^{-1} c(d g) c\left(\partial_{i}\right) g^{-1} c(d g) c\left(\partial_{j}\right) \\
& +\frac{1}{2} g^{-1} c(d g) c\left(\partial_{k}\right) \Gamma^{k} .
\end{aligned}
$$

Since $E$ is globally defined, we can compute it in the normal coordinates. Then, we have

$$
\begin{aligned}
& \operatorname{Tr}(E)\left(x_{0}\right) \\
& =\operatorname{Tr}\left[-\frac{s}{4}-\frac{1}{2} \partial_{j}\left(g^{-1} c(d g)\right) c\left(\partial_{j}\right)\right. \\
& \left.\quad-\frac{1}{4} g^{-1} c(d g) c\left(\partial_{i}\right) g^{-1} c(d g) c\left(\partial_{i}\right)\right]\left(x_{0}\right), \\
& \operatorname{Tr}\left[g^{-1} c(d g) c\left(\partial_{i}\right) g^{-1} c(d g) c\left(\partial_{i}\right)\right]\left(x_{0}\right) \\
& =g^{-2} \operatorname{Tr}\left[\sum_{i, k, l} \frac{\partial g}{\partial x_{k}} \frac{\partial g}{\partial x_{l}} c\left(\partial_{k}\right) c\left(\partial_{i}\right) c\left(\partial_{l}\right) c\left(\partial_{i}\right)\right]
\end{aligned}
$$

$$
\begin{gathered}
=g^{-2} \operatorname{Tr}\left[\sum_{i, k}\left(\frac{\partial g}{\partial x_{k}}\right)^{2} c\left(\partial_{k}\right) c\left(\partial_{i}\right) c\left(\partial_{k}\right) c\left(\partial_{i}\right)\right] \\
=g^{-2} \operatorname{Tr}\left[\sum_{i \neq k}\left(\frac{\partial g}{\partial x_{k}}\right)^{2} c\left(\partial_{k}\right) c\left(\partial_{i}\right) c\left(\partial_{k}\right) c\left(\partial_{i}\right)\right. \\
\left.+\sum_{k}\left(\frac{\partial g}{\partial x_{k}}\right)^{2} c\left(\partial_{k}\right)^{4}\right] \\
=-2 g^{-2} \sum_{k}\left(\frac{\partial g}{\partial x_{k}}\right)^{2} \operatorname{Tr}[I d] .
\end{gathered}
$$

Similarly,

$$
\operatorname{Tr}\left[\partial_{j}\left(g^{-1} c(d g)\right) c\left(\partial_{j}\right)\right]=\sum_{j}\left[\frac{1}{g^{2}}\left(\frac{\partial g}{\partial x_{j}}\right)^{2}-g^{-1} \frac{\partial^{2} g}{\partial x_{j}^{2}}\right] .
$$

So,

$$
\operatorname{Tr}\left[\frac{s}{6}+E\right]=-\frac{s}{3}+2 g^{-1} \sum_{j} \frac{\partial^{2} g}{\partial x_{j}^{2}}=-\frac{s}{3}-2 g^{-1} \Delta(g) .
$$

By

$$
\int_{M} f \Delta(g) d v o l_{M}=\int_{M}\langle d f, d g\rangle d v o l_{M},
$$

we get the following.

Theorem 17. Let $M$ be a 4-dimensional compact spin manifold without boundary; then

$$
\text { Wres }\left[f D^{-1} g D^{-1}\right]=-4 \pi^{2} \int_{M}\left[\frac{f g s}{3}+2\langle d f, d g\rangle\right] d v o l_{M} \text {. }
$$

Remark 18. In Theorem 17, when $f=g=e^{-2 h}$, we get a Kastler-Kalau-Walze theorem for conformal Dirac operators. In fact, Theorem 17 holds true for any choice of the smooth functions $f$ and $g$, since we can prove (61) by means of the symbolic calculus of pseudodifferential operators without using (55), and it is not essential that $f$ and $g$ are nowhere vanishing.

Now, we consider manifolds with boundary and we will compute $\widehat{\text { Wres }}\left[\pi^{+}\left(f D^{-1}\right) \pi^{+}\left(g D^{-1}\right)\right]$. As in [15], we have five terms as follows:

$$
\begin{array}{r}
\operatorname{term} \text { (a) (I) }=-f g \int_{\left|\xi^{\prime}\right|=1} \int_{-\infty}^{+\infty} \sum_{|\alpha|=1} \operatorname{trace}\left[\partial_{\xi^{\prime}}^{\alpha} \pi_{\xi_{n}}^{+} q_{-1}\right. \\
\left.\times \partial_{x^{\prime}}^{\alpha} \partial_{\xi_{n}} q_{-1}\right] \\
\times\left(x_{0}\right) d \xi_{n} \sigma\left(\xi^{\prime}\right) d x^{\prime}
\end{array}
$$




$$
\begin{aligned}
&-f \sum_{j<n} \partial_{j}(g) \int_{\left|\xi^{\prime}\right|=1} \int_{-\infty}^{+\infty} \sum_{|\alpha|=1} \operatorname{trace} {\left[\partial_{\xi_{j}} \pi_{\xi_{n}}^{+}\right.} \\
& \times q_{-1} \partial_{\xi_{n}} \\
&\left.\times q_{-1}\right] \\
& \times\left(x_{0}\right) d \xi_{n} \sigma\left(\xi^{\prime}\right) d x^{\prime}
\end{aligned}
$$

$\operatorname{term}(\mathrm{a})(\mathrm{II})=-\frac{1}{2} f g \int_{\left|\xi^{\prime}\right|=1} \int_{-\infty}^{+\infty} \operatorname{trace}\left[\partial_{x_{n}} \pi_{\xi_{n}}^{+} q_{-1}\right.$

$$
\begin{gathered}
\left.\times \partial_{\xi_{n}}^{2} q_{-1}\right] \\
\times\left(x_{0}\right) d \xi_{n} \sigma\left(\xi^{\prime}\right) d x^{\prime} \\
-\frac{1}{2} g \partial_{x_{n}} f \\
\times \int_{\left|\xi^{\prime}\right|=1} \int_{-\infty}^{+\infty} \operatorname{trace}\left[\pi_{\xi_{n}}^{+} q_{-1}\right. \\
\left.\times \partial_{\xi_{n}}^{2} q_{-1}\right] \\
\times\left(x_{0}\right) d \xi_{n} \sigma\left(\xi^{\prime}\right) d x^{\prime} \\
=-\frac{3}{8} \pi h^{\prime}(0) \Omega_{3} f g d x^{\prime} \\
-\frac{\pi i}{2} \Omega_{3} g \partial_{x_{n}}(f) d x^{\prime},
\end{gathered}
$$

term (a) (III) $=\frac{3}{8} \pi h^{\prime}(0) \Omega_{3} f g d x^{\prime}$

$$
+\frac{\pi i}{2} \Omega_{3} f \partial_{x_{n}}(g) d x^{\prime}
$$

As in [15], we have

$$
\begin{array}{r}
\operatorname{term}(\mathrm{b})=-i \int_{\left|\xi^{\prime}\right|=1} \int_{-\infty}^{+\infty} \operatorname{trace}[ \\
\pi_{\xi_{n}}^{+} q_{-2} \\
\left.\times \partial_{\xi_{n}} q_{-1}\right] \\
\times\left(x_{0}\right) d \xi_{n} \sigma\left(\xi^{\prime}\right) d x^{\prime} \\
=\frac{9}{8} f g \pi h^{\prime}(0) \Omega_{3} d x^{\prime}, \\
\text { term }(\mathrm{c})=-i \int_{\left|\xi^{\prime}\right|=1} \int_{-\infty}^{+\infty} \operatorname{trace}\left[\pi_{\xi_{n}}^{+} q_{-1}\right. \\
\left.\times \partial_{\xi_{n}} q_{-2}\right] \\
=-\frac{9}{8} f g \pi h^{\prime}(0) \Omega_{3} d x^{\prime} .
\end{array}
$$

So, the sum of terms (b) and (c) is zero. Then, we obtain

$$
\int_{\partial M} \Phi=\frac{\pi i \Omega_{3}}{2} \int_{\partial M}\left[f \partial_{x_{n}}(g)-g \partial_{x_{n}}(f)\right]_{x_{n}=0} d v o l_{\partial M}
$$

By the definition of the noncommutative residue for manifolds with boundary, we have that the interior term of $\widetilde{\text { Wres }}\left[\pi^{+}\left(f D^{-1}\right) \pi^{+}\left(g D^{-1}\right)\right]$ equals Wres $\left[f D^{-1} g D^{-1}\right]$. Then, by Theorem 17 and (64), we get the following.

Theorem 19. Let $M$ be a 4-dimensional compact spin manifold with boundary. Then,

$$
\begin{aligned}
& \overparen{\text { Wres }}\left[\pi^{+}\left(f D^{-1}\right) \pi^{+}\left(g D^{-1}\right)\right] \\
& =-4 \pi^{2} \int_{M}\left[\frac{f g s}{3}+2\langle d f, d g\rangle\right] d v o l_{M} \\
& \quad+\left.\frac{\pi i \Omega_{3}}{2} \int_{\partial M}\left[f \partial_{x_{n}}(g)-g \partial_{x_{n}}(f)\right]\right|_{x_{n}=0} d v o l_{\partial M} .
\end{aligned}
$$

When $f=1$ and $g=x_{n} K$ near the boundary, we have that the boundary term is proportional to the gravitational action on the boundary.

\section{The Spectral Action for Perturbations of Dirac Operators}

In [18], Iochum and Levy computed heat kernel coefficients for Dirac operators with one-form perturbations and proved that there are no tadpoles for compact spin manifolds without boundary. In [17], they investigated the spectral action for scalar perturbations of Dirac operators. In [19], Hanisch et al. derived a formula for the gravitational part of the spectral action for Dirac operators on 4-dimensional spin manifolds with totally antisymmetric torsion. In fact, Dirac operators with totally antisymmetric torsion are three-form perturbations of Dirac operators. In this section, we will give some details on the spectral action for Dirac operators with scalar perturbations. We also compute the spectral action for Dirac operators with two-form perturbations on 4-dimensional compact spin manifolds without boundary.

For the perturbed self-adjoint Dirac operator $D_{\Psi}$, we will calculate the bosonic part of the spectral action. It is defined to be the number of eigenvalues of $D_{\Psi}$ in the interval $[-\wedge, \wedge]$ with $\wedge \in \mathbf{R}^{+}$. It is expressed as

$$
I=\operatorname{Tr} F\left(\frac{D_{\Psi}^{2}}{\wedge^{2}}\right)
$$

Here, $T r$ denotes the operator trace in the $L^{2}$ completion of $\Gamma(M, S(T M))$ and $F: \mathbf{R}^{+} \rightarrow \mathbf{R}^{+}$is a cut-off function with support in the interval $[0,1]$ which is constant near the origin. Let $\operatorname{dim} M=n$. By Lemma 1.7.4 in [22], we have the heat trace asymptotics, for $t \rightarrow 0$,

$$
\operatorname{Tr}\left(e^{-t D_{\Psi}^{2}}\right) \sim \sum_{m \geq 0} t^{m-n / 2} a_{2 m}\left(D_{\Psi}^{2}\right) .
$$


One uses the Seeley-DeWitt coefficients $a_{2 m}\left(D_{\Psi}^{2}\right)$ and $t=$ $\wedge^{-2}$ to obtain an asymptotics for the spectral action when $\operatorname{dim} M=4$

$$
\begin{aligned}
I= & \operatorname{tr} F\left(\frac{D_{\Psi}^{2}}{\wedge^{2}}\right) \sim \wedge^{4} F_{4} a_{0}\left(D_{\Psi}^{2}\right) \\
& +\wedge^{2} F_{2} a_{2}\left(D_{\Psi}^{2}\right)+\wedge^{0} F_{0} a_{4}\left(D_{\Psi}^{2}\right) \quad \text { as } \wedge \longrightarrow \infty
\end{aligned}
$$

with the first three moments of the cut-off function which are given by $F_{4}=\int_{0}^{\infty} s F(s) d s, F_{2}=\int_{0}^{\infty} F(s) d s$, and $F_{0}=F(0)$. Let

$$
\Omega_{i j}=\nabla_{e_{i}} \nabla_{e_{j}}-\nabla_{e_{j}} \nabla_{e_{i}}-\nabla_{\left[e_{i}, e_{j}\right]} \cdot
$$

We use [22, Thm 4.1.6] to obtain the first three coefficients of the heat trace asymptotics:

$$
\begin{gathered}
a_{0}\left(D_{\Psi}\right)=(4 \pi)^{-n / 2} \int_{M} \operatorname{Tr}(I d) d v o l, \\
a_{2}\left(D_{\Psi}\right)=(4 \pi)^{-n / 2} \int_{M} \operatorname{Tr}\left[\frac{s}{6}+E\right] d v o l, \\
a_{4}\left(D_{\Psi}\right) \\
=\frac{(4 \pi)^{-n / 2}}{360} \\
\times \int_{M} \operatorname{Tr}\left[-12 R_{i j i j, k k}+5 R_{i j i j} R_{k l k l}\right. \\
\quad-2 R_{i j i k} R_{l j l k}+2 R_{i j k l} R_{i j k l}-60 R_{i j i j} E \\
\left.\quad+180 E^{2}+60 E_{, k k}+30 \Omega_{i j} \Omega_{i j}\right] d v o l .
\end{gathered}
$$

When $\Psi=f$, by (15) and (71),

$$
\begin{aligned}
a_{2}\left(D_{f}\right)=(2 \pi)^{-n / 2}[ & \left.-\frac{s}{12}+(n-1) f^{2}\right], \\
5 s^{2}+60 s E+180 E^{2}= & \frac{5}{4} s^{2}-30(n-1) s f^{2} \\
& +180(n-1)^{2} f^{4} .
\end{aligned}
$$

$\operatorname{Tr}\left[\Omega_{i j} \Omega_{i j}\right]$ is globally defined; thus we only compute it in normal coordinates about $x_{0}$ and the local orthonormal frame $e_{i}$ obtained by parallel transport along geodesics from $x_{0}$. Then,

$$
\omega_{s t}\left(x_{0}\right)=0, \quad \partial_{i}\left(c\left(e_{j}\right)\right)=0, \quad\left[e_{i}, e_{j}\right]\left(x_{0}\right)=0 .
$$

We know that the curvature of the canonical spin connection is

$$
R^{S}\left(e_{i}, e_{j}\right)=-\frac{1}{4} \sum_{s, t=1}^{n} R_{i j s t}^{M} c\left(e_{s}\right) c\left(e_{t}\right)
$$

Then, we have

$$
\begin{aligned}
& \Omega\left(e_{i}, e_{j}\right)\left(x_{0}\right) \\
& =\left[e_{i}+\frac{1}{4} \sum_{s, t} \omega_{s t}\left(e_{i}\right) c\left(e_{s}\right) c\left(e_{t}\right)-f c\left(e_{i}\right)\right] \\
& \quad \times\left[e_{j}+\frac{1}{4} \sum_{s, t} \omega_{s t}\left(e_{j}\right) c\left(e_{s}\right) c\left(e_{t}\right)-f c\left(e_{j}\right)\right] \\
& -\left[e_{j}+\frac{1}{4} \sum_{s, t} \omega_{s t}\left(e_{j}\right) c\left(e_{s}\right) c\left(e_{t}\right)-f c\left(e_{i}\right)\right] \\
& \quad \times\left[e_{i}+\frac{1}{4} \sum_{s, t} \omega_{s t}\left(e_{i}\right) c\left(e_{s}\right) c\left(e_{t}\right)-f c\left(e_{i}\right)\right] \\
& =-\frac{1}{4} \sum_{s, t=1}^{n} R_{i j s t}^{M} c\left(e_{s}\right) c\left(e_{t}\right)-e_{i}(f) c\left(e_{j}\right) \\
& \quad+e_{j}(f) c\left(e_{i}\right)+2 f^{2} c\left(e_{i}\right) c\left(e_{j}\right), \quad \text { for } i \neq j .
\end{aligned}
$$

So,

$$
\begin{aligned}
& \operatorname{Tr}\left[\Omega_{i j} \Omega_{i j}\right]\left(x_{0}\right) \\
&=\sum_{i \neq j} \operatorname{Tr}\left\{\frac{1}{16} \sum_{s, t, s_{1}, t_{1}=1}^{n} R_{i j s t}^{M} R_{i j s_{1} t_{1}}^{M} c\left(e_{s}\right)\right. \\
& \quad \times c\left(e_{t}\right) c\left(e_{s_{1}}\right) c\left(e_{t_{1}}\right) \\
&+e_{i}(f)^{2} c\left(e_{j}\right)^{2}+e_{j}(f)^{2} c\left(e_{i}\right)^{2} \\
&+4 f^{4} c\left(e_{i}\right) c\left(e_{j}\right) c\left(e_{i}\right) c\left(e_{j}\right) \\
& \quad-\frac{f^{2}}{2} \sum_{s, t=1}^{n} R_{i j s t}^{M}\left[c\left(e_{s}\right) c\left(e_{t}\right) c\left(e_{i}\right) c\left(e_{j}\right)\right. \\
&\left.\quad+c\left(e_{i}\right) c\left(e_{j}\right) c\left(e_{s}\right) c\left(e_{t}\right)\right] \\
&\left.-e_{i}(f) e_{j}(f)\left[c\left(e_{j}\right) c\left(e_{i}\right)+c\left(e_{i}\right) c\left(e_{j}\right)\right]\right\} .
\end{aligned}
$$

By (21), we obtain

$$
\begin{gathered}
\sum_{i, j, s, t, s_{1}, t_{1}=1}^{n} \operatorname{Tr}\left[\frac{1}{16} R_{i j s t}^{M} R_{i j s_{1} t_{1}}^{M} c\left(e_{s}\right) c\left(e_{t}\right) c\left(e_{s_{1}}\right) c\left(e_{t_{1}}\right)\right] \\
=-\frac{d}{8} \sum_{i, j, s, t=1}^{n}\left(R_{i j s t}^{M}\right)^{2}, \\
\sum_{i \neq j} \operatorname{Tr}\left[e_{i}(f)^{2} c\left(e_{j}\right)^{2}+e_{j}(f)^{2} c\left(e_{i}\right)^{2}\right] \\
=2 d(1-n) \sum_{i} e_{i}(f)^{2}=2 d(1-n)|d f|^{2},
\end{gathered}
$$




$$
\begin{gathered}
\sum_{i \neq j} \operatorname{Tr}\left[4 f^{4} c\left(e_{i}\right) c\left(e_{j}\right) c\left(e_{i}\right) c\left(e_{j}\right)\right]=-4 d n(n-1) f^{4}, \\
\sum_{i \neq j} \operatorname{Tr}\left[-e_{i}(f) e_{j}(f)\left(c\left(e_{j}\right) c\left(e_{i}\right)+c\left(e_{i}\right) c\left(e_{j}\right)\right)\right]=0, \\
\sum_{i \neq j} \operatorname{Tr}\left\{-\frac{f^{2}}{2} R_{i j s t}^{M}\left[c\left(e_{s}\right) c\left(e_{t}\right) c\left(e_{i}\right) c\left(e_{j}\right)\right.\right. \\
\left.\left.+c\left(e_{i}\right) c\left(e_{j}\right) c\left(e_{s}\right) c\left(e_{t}\right)\right]\right\} \\
=-2 f^{2} d s .
\end{gathered}
$$

By (78)-(83), we obtain

$$
\begin{aligned}
\operatorname{Tr}\left[\Omega_{i j} \Omega_{i j}\right]= & -\frac{d}{8}\left(R_{i j s t}^{M}\right)^{2}+2 d(1-n)|d f|^{2} \\
& -2 f^{2} d s-4 d n(n-1) f^{4} .
\end{aligned}
$$

By (72), (74), and (84), we get the following.

Proposition 20 (see [17]). The following equality holds:

$$
\begin{aligned}
a_{4}\left(D_{f}\right)= & \frac{d}{360 \times(4 \pi)^{n / 2}} \\
& \times\left[3 \Delta s+\frac{5}{4} s^{2}-30(n+1) s f^{2}\right. \\
& +60(n-1)(n-3) f^{4} \\
& -2 R_{i j i k} R_{l j l k}-\frac{7}{4} R_{i j s t}^{2} \\
& \left.+60(1-n)|d f|^{2}-60(n-1) \Delta\left(f^{2}\right)\right] .
\end{aligned}
$$

In the following, we assume that $\operatorname{dim} M=4$ and $d=4$. We let $\Psi$ be a two-form; namely, $\Psi=\sum_{k, l} a_{k l} e^{k} \wedge e^{l}$, where $a_{k l}=-a_{l k}$. We may consider $\sqrt{-1} \Psi$ for self-adjoint perturbed Dirac operators. By Corollary 4 , we obtain

$$
\begin{aligned}
a_{2}\left(D_{\Psi}\right) & =d(4 \pi)^{-n / 2}\left[-\frac{s}{12}+(6-2 n)|\Psi|^{2}\right] \\
& =\frac{-1}{4 \pi^{2}}\left[\frac{s}{12}+2|\Psi|^{2}\right] .
\end{aligned}
$$

Firstly, we compute $\operatorname{Tr}\left(E^{2}\right)$. By (20) and (75),

$$
\begin{gathered}
\sum_{j, k, l} e_{j}\left(a_{k l}\right)\left[c\left(e_{k}\right) c\left(e_{l}\right) c\left(e_{j}\right)-c\left(e_{j}\right) c\left(e_{k}\right) c\left(e_{l}\right)\right] \\
=4 e_{k}\left(a_{k l}\right) c\left(e_{l}\right) \\
a_{k l}\left[c\left(e_{i}\right) c\left(e_{k}\right) c\left(e_{l}\right)+c\left(e_{k}\right) c\left(e_{l}\right) c\left(e_{i}\right)\right] \\
\times a_{k_{1} l_{1}}\left[c\left(e_{i}\right) c\left(e_{k_{1}}\right) c\left(e_{l_{1}}\right)\right. \\
\left.+c\left(e_{k_{1}}\right) c\left(e_{l_{1}}\right) c\left(e_{i}\right)\right]
\end{gathered}
$$

$$
\begin{aligned}
= & \sum_{i \neq k, l} 2 a_{k l} c\left(e_{k}\right) c\left(e_{l}\right) c\left(e_{i}\right) \\
& \times \sum_{i \neq k_{1}, l_{1}} 2 a_{k_{1} l_{1}} c\left(e_{i}\right) c\left(e_{k_{1}}\right) c\left(e_{l_{1}}\right) \\
= & -4 \sum_{i \neq k, l, k_{1}, l_{1}} a_{k l} a_{k_{1} l_{1}} c\left(e_{k}\right) \\
& \times c\left(e_{l}\right) c\left(e_{k_{1}}\right) c\left(e_{l_{1}}\right) \\
= & -4\left(\sum_{k=k_{1}, l \neq l_{1}}+\sum_{k=l_{1}, l \neq k_{1}}+\sum_{l=k_{1}, k \neq l_{1}}\right.
\end{aligned}
$$

$$
\begin{gathered}
\left.+\sum_{l=l_{1}, k \neq k_{1}}+\sum_{k=k_{1}, l=l_{1}}+\sum_{k=l_{1}, l=k_{1}}\right) \\
\times\left[a_{k l} a_{k_{1} l_{1}} c\left(e_{k}\right) c\left(e_{l}\right) c\left(e_{k_{1}}\right) c\left(e_{l_{1}}\right)\right] \\
=-16 \sum_{l \neq l_{1}} a_{k l} a_{k l_{1}} c\left(e_{l}\right) c\left(e_{l_{1}}\right)+16 a_{k l}^{2} .
\end{gathered}
$$

Similar to (88), we have

$$
\begin{aligned}
& {\left[a_{k l} c\left(e_{k}\right) c\left(e_{l}\right)\right]^{2}} \\
& =\sum_{k \neq l \neq k_{1} \neq l_{1}} a_{k l} a_{k_{1} l_{1}} c\left(e_{k}\right) c\left(e_{l}\right) c\left(e_{k_{1}}\right) c\left(e_{l_{1}}\right) \\
& \quad+4 \sum_{l \neq l_{1}} a_{k l} a_{k l_{1}} c\left(e_{l}\right) c\left(e_{l_{1}}\right)-2 a_{k l}^{2} .
\end{aligned}
$$

Then,

$$
\begin{aligned}
E= & -\frac{s}{4}+2 e_{k}\left(a_{k l}\right) c\left(e_{l}\right)-\sum_{k \neq l \neq k_{1} \neq l_{1}} a_{k l} a_{k_{1} l_{1}} \\
& \times c\left(e_{k}\right) c\left(e_{l}\right) c\left(e_{k_{1}}\right) c\left(e_{l_{1}}\right)-2 a_{k l}^{2} .
\end{aligned}
$$

Now, we can compute $\operatorname{Tr}\left(E^{2}\right)$. Consider

$$
\begin{aligned}
\operatorname{Tr} & {\left[2 e_{k}\left(a_{k l}\right) c\left(e_{l}\right) 2 e_{k_{1}}\left(a_{k_{1} l_{1}}\right) c\left(e_{l_{1}}\right)\right] } \\
& =-4 d e_{k}\left(a_{k l}\right) e_{k_{1}}\left(a_{k_{1} l}\right)=-4|\delta \Psi|^{2}\left(x_{0}\right),
\end{aligned}
$$

where $\delta$ is the adjoint operator of $d$. We have

$$
\begin{array}{r}
\operatorname{Tr}\left[\left(-\frac{s}{4}-2|\Psi|^{2}\right)^{2}\right]=4 \\
\sum_{k \neq l \neq k_{1} \neq l_{1}} \sum_{p \neq q \neq r \neq t} \operatorname{Tr}\left[a_{k l} a_{k_{1} l_{1}} c\left(e_{k}\right) c\left(e_{l}\right) c\left(e_{k_{1}}\right)\right. \\
\times c\left(e_{l_{1}}\right) a_{p q} a_{r t} c\left(e_{p}\right) \\
\left.\times c\left(e_{q}\right) c\left(e_{r}\right) c\left(e_{t}\right)\right]
\end{array}
$$




$$
\begin{aligned}
& =\sum_{\left\{k, l, k_{1}, l_{1}\right\}=\{p, q, r, t\}} \operatorname{Tr}\left[a_{k l} a_{k_{1} l_{1}} c\left(e_{k}\right) c\left(e_{l}\right)\right. \\
& \times c\left(e_{k_{1}}\right) c\left(e_{l_{1}}\right) a_{p q} a_{r t} \\
& \left.\times c\left(e_{p}\right) c\left(e_{q}\right) c\left(e_{r}\right) c\left(e_{t}\right)\right] \\
& =8 d|\Psi|^{4}-16 d a_{k l} a_{k_{1} l_{1}} a_{k k_{1}} a_{l l_{1}} \\
& =32|\Psi|^{4}-4 i_{e_{k}} i_{e_{l}}(\Psi) i_{e_{k_{1}}} i_{e_{l_{1}}} \\
& \times(\Psi) i_{e_{k}} i_{e_{k_{1}}}(\Psi) i_{e_{l}} i_{e_{l_{1}}}(\Psi) .
\end{aligned}
$$

By (90)-(93), we get

$$
\begin{aligned}
\operatorname{Tr}\left(E^{2}\right)=4\left[-|\delta \Psi|^{2}+\frac{s^{2}}{16}+s|\Psi|^{2}+12|\Psi|^{4}\right. & \\
& \left.\quad-i_{e_{k}} i_{e_{l}}(\Psi) i_{e_{k_{1}}} i_{e_{l_{1}}}(\Psi) i_{e_{k}} i_{e_{k_{1}}}(\Psi) i_{e_{l}} i_{e_{l_{1}}}(\Psi)\right] .
\end{aligned}
$$

In the following, we compute $\operatorname{Tr}\left[\Omega\left(e_{i}, e_{j}\right) \Omega\left(e_{i}, e_{j}\right)\right]$. By (12), we have

$$
\begin{aligned}
\Omega\left(e_{i}, e_{j}\right)= & R^{S(T M)}\left(e_{i}, e_{j}\right) \\
& -\frac{1}{4} \nabla_{e_{i}}^{S(T M)}\left(c\left(e_{j}\right) \Psi+\Psi c\left(e_{j}\right)\right) \\
& +\frac{1}{4} \nabla_{e_{j}}^{S(T M)}\left(c\left(e_{i}\right) \Psi+\Psi c\left(e_{i}\right)\right) \\
& +\frac{1}{4}\left[c\left(\left[e_{i}, e_{j}\right]\right) \Psi+\Psi_{c}\left(\left[e_{i}, e_{j}\right]\right)\right] .
\end{aligned}
$$

Since $\nabla^{S(T M)}$ is a Clifford connection and $\nabla^{T M}$ has no torsion, we get

$$
\begin{aligned}
\Omega\left(e_{i}, e_{j}\right)= & R^{S(T M)}\left(e_{i}, e_{j}\right)-\frac{1}{4} \nabla_{e_{i}}^{S(T M)}(\Psi) c\left(e_{j}\right) \\
& -\frac{1}{4} c\left(e_{j}\right) \nabla_{e_{i}}^{S(T M)}(\Psi) \\
& +\frac{1}{4} \nabla_{e_{j}}^{S(T M)}(\Psi) c\left(e_{i}\right)+\frac{1}{4} c\left(e_{i}\right) \nabla_{e_{j}}^{S(T M)}(\Psi) .
\end{aligned}
$$

Since the trace of the odd Clifford elements is zero and

$$
\begin{gathered}
\operatorname{Tr}\left[\sum_{i, j=1}^{n}\left(\frac{1}{4} \sum_{s, t=1}^{n} R_{i j s t}^{M} c\left(e_{s}\right) c\left(e_{t}\right)\right)^{2}\right] \\
=-\frac{1}{2} \sum_{i, j, s, t=1}^{n} R_{i j s t}^{2},
\end{gathered}
$$

we get

$$
\begin{gathered}
\operatorname{Tr} \sum_{i, j=1}^{n}\left[\Omega_{i, j}^{2}\right] \\
=-\frac{1}{2} \sum_{i, j, s, t=1}^{n} R_{i j s t}^{2} \\
+\frac{1}{16} \operatorname{Tr}\left[\sum _ { i , j = 1 } ^ { n } \left(-\nabla_{e_{i}}^{S(T M)}(\Psi) c\left(e_{j}\right)\right.\right. \\
\quad-c\left(e_{j}\right) \nabla_{e_{i}}^{S(T M)}(\Psi)+\nabla_{e_{j}}^{S(T M)}(\Psi) \\
\left.\left.\quad \times c\left(e_{i}\right)+c\left(e_{i}\right) \nabla_{e_{j}}^{S(T M)}(\Psi)\right)^{2}\right] .
\end{gathered}
$$

By (72), (94), and (98), we obtain the following.

Proposition 21. Let $\Psi$ be a two-form and let $M$ be a 4dimensional compact spin manifold without boundary. Then,

$$
\begin{aligned}
& a_{4}\left(D_{\Psi}\right)=\frac{1}{1440 \pi^{2}}\{ \Delta\left(3 s+120|\Psi|^{2}\right)+\frac{5}{4} s^{2}-2 \sum_{i, l, j, k=1}^{n} R_{i j i k} \\
& \times R_{l j l k}-\frac{7}{4} \sum_{i, j, k, l=1}^{n} R_{i j k l}^{2}+60 s|\Psi|^{2} \\
&-180|\delta \Psi|^{2}+2160|\Psi|^{4} \\
&-180 \sum_{k, l, k_{1}, l_{1}=1}^{n} i_{e_{k}} i_{e_{l}} \\
& \times(\Psi) i_{e_{k_{1}}} i_{e_{l_{1}}}(\Psi) i_{e_{k}} i_{e_{k_{1}}}(\Psi) i_{e_{l}} i_{e_{1}}(\Psi) \\
&+\frac{15}{8} \operatorname{Tr}_{\sum_{i, j=1}^{n}\left(-\nabla_{e_{i}}^{S(T M)}(\Psi) c\left(e_{j}\right)\right.} \\
&-c\left(e_{j}\right) \nabla_{e_{i}}^{S(T M)}(\Psi) \\
&+\nabla_{e_{j}}^{S(T M)}(\Psi) c\left(e_{i}\right) \\
&+c\left(e_{i}\right) \nabla_{e_{j}}^{S(T M)}
\end{aligned}
$$

Remark 22. In fact, Proposition 21 holds true, under analogous hypotheses for manifolds of arbitrary dimension and a general two-form $\Psi$ after revising some coefficients.

\section{Conflict of Interests}

The author declares that there is no conflict of interests regarding the publication of this paper. 


\section{Acknowledgments}

This work was supported by NCET-13-0721 and NSFC no. 11271062. The author would like to thank the referees for their careful reading and helpful comments. He also thanks Professor Huitao Feng for his helpful comments.

\section{References}

[1] V. Guillemin, "A new proof of Weyl's formula on the asymptotic distribution of eigenvalues," Advances in Mathematics, vol. 55, no. 2, pp. 131-160, 1985.

[2] M. Wodzicki, "Noncommutative residue. Chapter I. Fundamentals," in K-Theory, Arithmetic and Geometry, Lecture Notes in Mathematics, pp. 320-399, 1987.

[3] A. Connes, "Quantized calculus and applications," in 11th International Conference on Mathematical P Conference, pp. 1536, Internat Press, Cambridge, Mass, USA, 1995.

[4] A. Connes, "The action functional in noncommutative geometry," Communications in Mathematical Physics, vol. 117, no. 4, pp. 673-683, 1988.

[5] D. Kastler, "The Dirac operator and gravitation," Communications in Mathematical Physics, vol. 166, no. 3, pp. 633-643, 1995.

[6] W. Kalau and M. Walze, "Gravity, non-commutative geometry and the Wodzicki residue," Journal of Geometry and Physics, vol. 16, no. 4, pp. 327-344, 1995.

[7] T. Ackermann, "A note on the Wodzicki residue," Journal of Geometry and Physics, vol. 20, no. 4, pp. 404-406, 1996.

[8] T. Ackermann and J. Tolksdorf, "A generalized Lichnerowicz formula, the Wodzicki residue and gravity," Journal of Geometry and Physics, vol. 19, no. 2, pp. 143-150, 1996.

[9] R. Ponge, "Noncommutative residue for Heisenberg manifolds: applications in CR and contact geometry," Journal of Functional Analysis, vol. 252, no. 2, pp. 399-463, 2007.

[10] U. Battisti and S. Coriasco, "A note on the Einstein-Hilbert action and Dirac operators on $R^{n}$," Journal of Pseudo-Differential Operators and Applications, vol. 2, no. 3, pp. 303-315, 2011.

[11] U. Battisti and S. Coriasco, "Wodzicki residue for operators on manifolds with cylindrical ends," Annals of Global Analysis and Geometry, vol. 40, no. 2, pp. 223-249, 2011.

[12] F. Nicola, "Trace functionals for a class of pseudo-differential operators in $R^{n}$," Mathematical Physics, Analysis and Geometry, vol. 6, no. 1, pp. 89-105, 2003.

[13] B. V. Fedosov, F. Golse, E. Leichtnam, and E. Schrohe, "The noncommutative residue for manifolds with boundary," Journal of Functional Analysis, vol. 142, no. 1, pp. 1-31, 1996.

[14] E. Schrohe, "Noncommutative residue, Dixmier's trace, and heat trace expansions on manifolds with boundary," Contemporary Mathematics, vol. 242, pp. 161-186, 1999.

[15] Y. Wang, "Gravity and the noncommutative residue for manifolds with boundary," Letters in Mathematical Physics, vol. 80, no. 1, pp. 37-56, 2007.

[16] Y. Wang, "Lower-dimensional volumes and Kastler-KalauWalze type theorem for manifolds with boundary," Communications in Theoretical Physics, vol. 54, pp. 38-42, 2010.

[17] A. Sitarz and A. Zajac, "Spectral action for scalar perturbations of Dirac operators," Letters in Mathematical Physics, vol. 98, no. 3, pp. 333-348, 2011.

[18] B. Iochum and C. Levy, "Tadpoles and commutative spectral triples," Journal of Noncommutative Geometry, vol. 5, no. 3, pp. 299-329, 2011.
[19] F. Hanisch, F. Pfäffle, and C. A. Stephan, "The spectral action for Dirac operators with skew-symmetric torsion," Communications in Mathematical Physics, vol. 300, no. 3, pp. 877-888, 2010.

[20] A. Connes and H. Moscovici, "Type III and spectral triples," in Traces in Number Theory, Geometry and Quantum Fields, Aspects of Mathematics E38, pp. 57-71, Vieweg, 2008.

[21] A. Connes and A. H. Chamseddine, "Inner fluctuations of the spectral action," Journal of Geometry and Physics, vol. 57, no. 1, pp. 1-21, 2006.

[22] P. B. Gilkey, Invariance Theory, the Heat Equation, and the Atiyah-Singer Index Theorem, vol. 11 of Mathematics Lecture Series, 1984. 


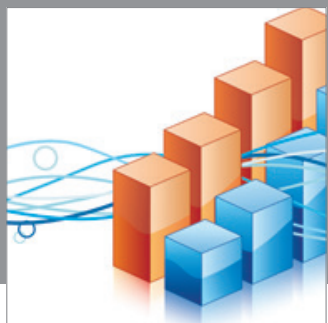

Advances in

Operations Research

mansans

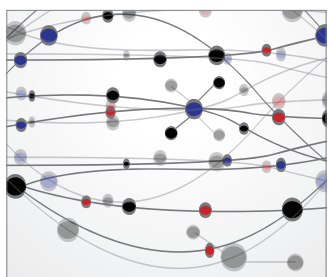

The Scientific World Journal
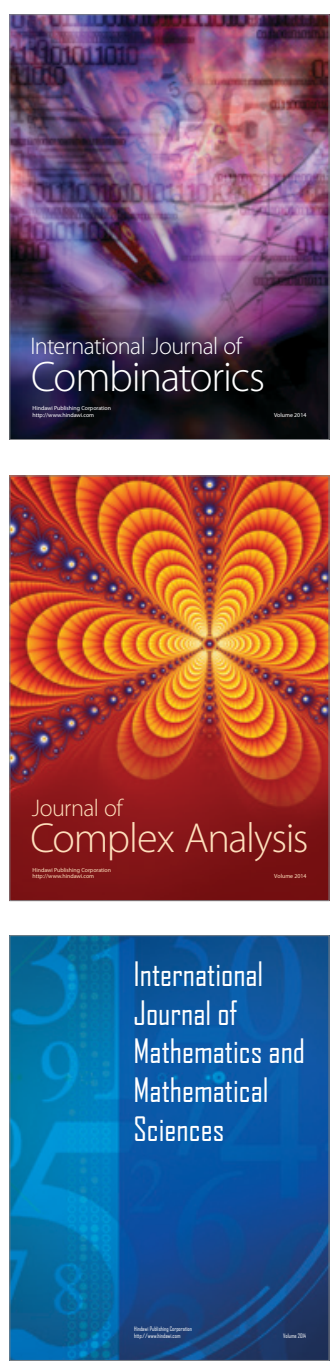
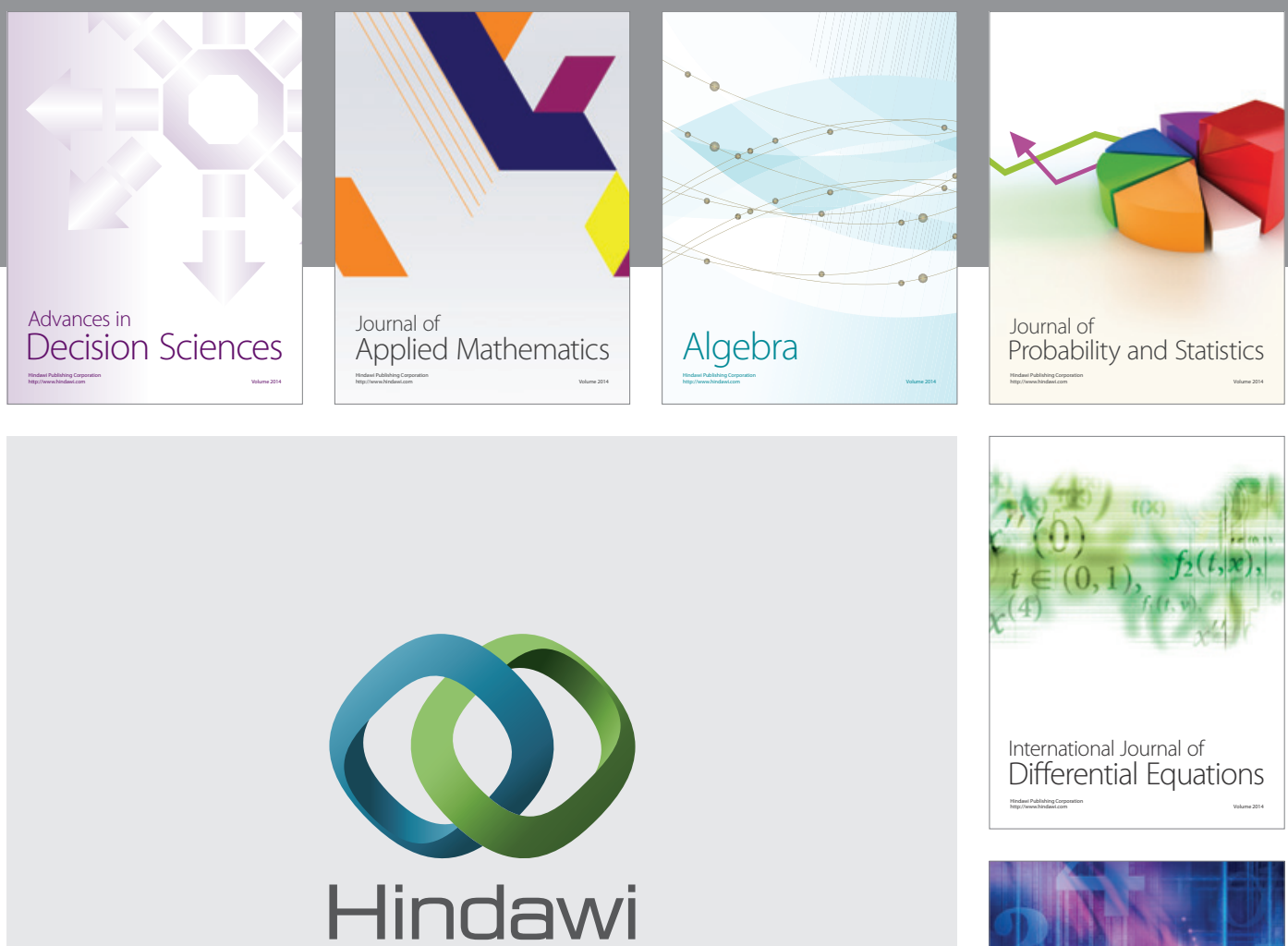

Submit your manuscripts at http://www.hindawi.com
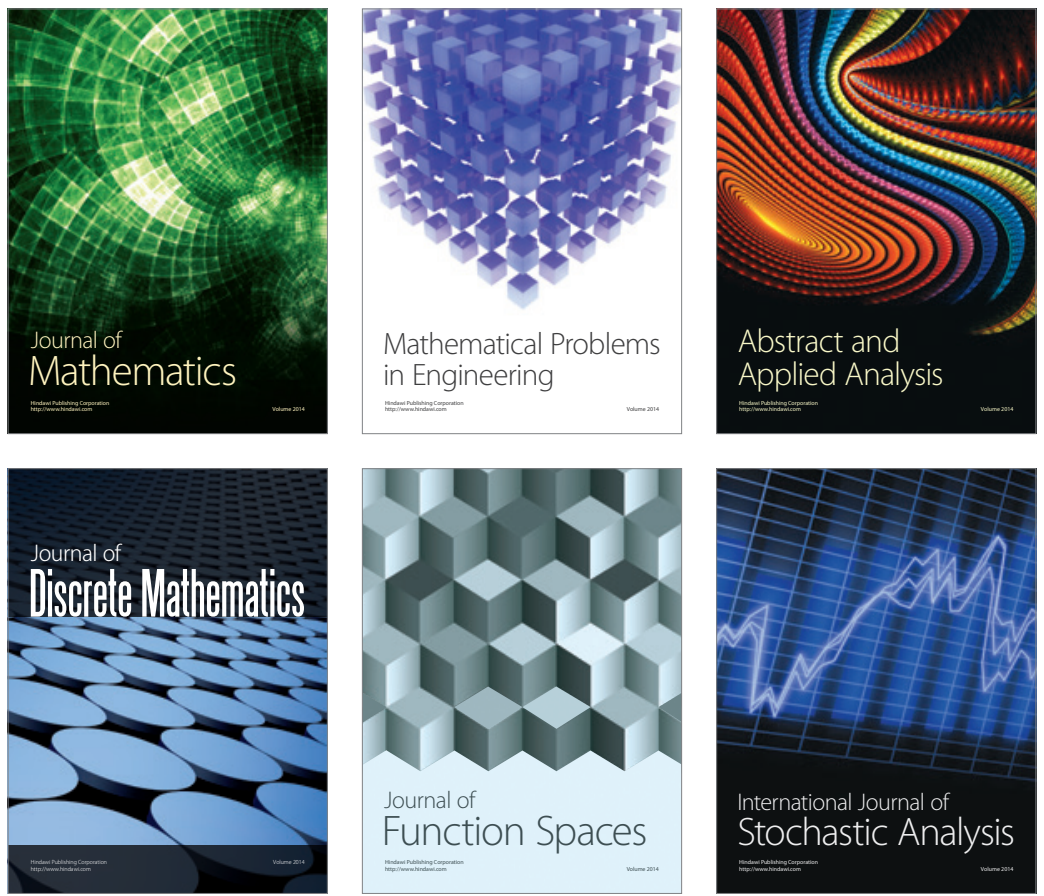

Journal of

Function Spaces

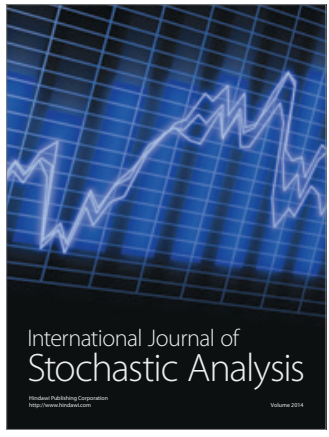

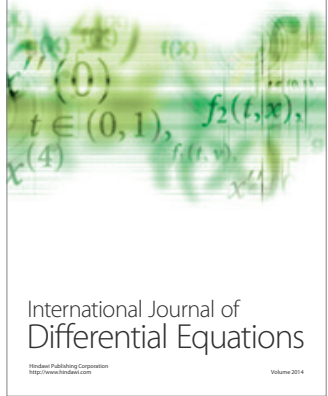
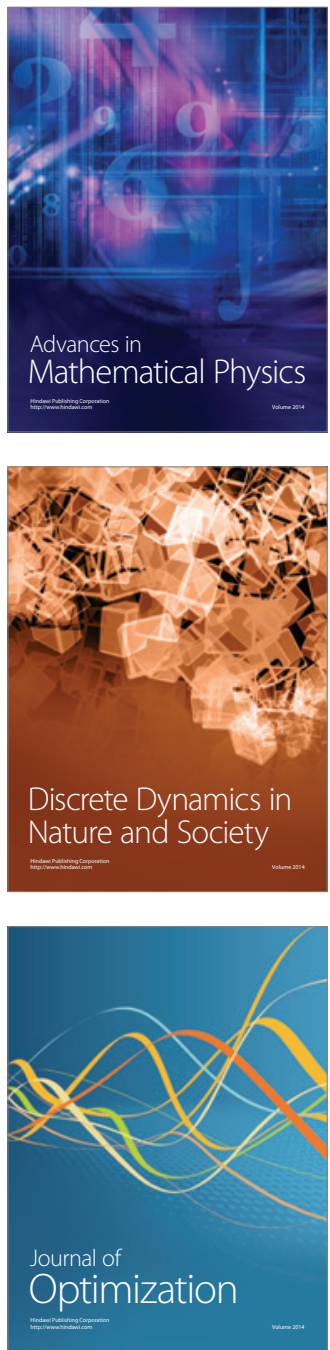